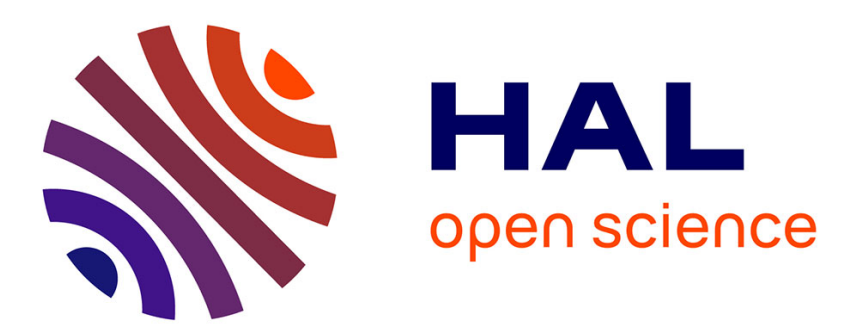

\title{
Derivation of a voltage density equation from a voltage-conductance kinetic model for networks of integrate-and-fire neurons.
}

Benoît Perthame, Delphine Salort

\section{- To cite this version:}

Benoît Perthame, Delphine Salort. Derivation of a voltage density equation from a voltageconductance kinetic model for networks of integrate-and-fire neurons.. Communications in Mathematical Sciences, 2019, 17 (5), 10.4310/CMS.2019.v17.n5.a2 . hal-01881950v2

\section{HAL Id: hal-01881950 \\ https://hal.sorbonne-universite.fr/hal-01881950v2}

Submitted on 4 Dec 2018

HAL is a multi-disciplinary open access archive for the deposit and dissemination of scientific research documents, whether they are published or not. The documents may come from teaching and research institutions in France or abroad, or from public or private research centers.
L'archive ouverte pluridisciplinaire $\mathbf{H A L}$, est destinée au dépôt et à la diffusion de documents scientifiques de niveau recherche, publiés ou non, émanant des établissements d'enseignement et de recherche français ou étrangers, des laboratoires publics ou privés. 


\title{
Derivation of an integrate\&fire equation for neural networks from a voltage-conductance kinetic model
}

\author{
Benoît Perthame* Delphine Salort ${ }^{\dagger}$
}

December 4, 2018

In memory of late David CAI, a pioneer in mathematical neuroscience

2010 Mathematics Subject Classification. 35B65, 35Q84, 62M45, 82C32, 92B20.

Keywords. Integrate-and-fire networks; Voltage-conductance Vlasov equation; Neural assemblies; Fokker-Planck kinetic equation; Slow-fast dynamics; Asymptotic analysis;

\begin{abstract}
In terms of mathematical structure, the voltage-conductance kinetic systems for neural networks can be compared to a kinetic equations with a macroscopic limit which turns out to be the Integrate and Fire equation. This article is devoted to mathematical study of the slow-fast limit of the kinetic type equation to an I\&F equation. After proving the weak convergence of the voltage-conductance kinetic problem to potential only I\&F equation, we study the main qualitative properties of the solution of the I\&F model, with respect to the strength of interconnections of the network. In particular, we obtain asymptotic convergence to a unique stationary state for weak connectivity regimes. For intermediate connectivities, we prove linear instability and numerically exhibit periodic solutions. These results about the I\&F model suggest that the more complex kinetic equation shares some similar dynamics.
\end{abstract}

\section{Introduction}

The voltage-conductance kinetic systems for neural networks are nonlinear $(2+1)$ dimensional kinetic Fokker-Plank equation which is established in [1, 2]. Based on neuro-physical concepts adapted in particular to the visual cortex, they describe the probability density $p_{\varepsilon}(v, g, t)$ to find neurons at time $t$ with a membrane potential $v \in\left(V_{R}, V_{E}\right)$ and a conductance $g>0$. Here, $V_{E}$ denotes the excitatory reversal potential and the reset potential satisfies $V_{R} \leq V_{L}$ with $V_{L}$ the leak potential. There are several variants of the equation depending on the physical interpretation of variable, see, e.g., [3, 4]. The mathematical structure of these systems is rather complex and has attracted the interest of mathematicians $[5,6]$. In particular difficulties related to boundary conditions and partial parabolicity make the system rather uneasy to handle and connect it to present interest about hypoellipticity in kinetic equations, see $[7,8,9]$ and the references therein. A rather striking finding in [5] is the numerical

\footnotetext{
*Sorbonne Université, Université Paris-Diderot, CNRS, INRIA, Laboratoire Jacques-Louis Lions, F-75005 Paris, France. Email : Benoit.Perthame@sorbonne-universite.fr

${ }^{\dagger}$ Sorbonne Université, CNRS, Laboratoire de Biologie Computationnelle et Quantitative, UMR 7238, F-75005 Paris, France. Email : delphine.salort@sorbonne-universite.fr
} 
observation of periodic solutions describing spontaneous activity of the network, a phenomena which is common to other neural assembly models (see $[10,11,12,13])$.

Here, and for the sake of analytical tractability, we consider a simplified version, with an integral absorption term rather than a boundary firing, which keeps the main structure of the system which is written

$$
\begin{aligned}
\frac{\partial}{\partial t} p_{\varepsilon}+\frac{\partial}{\partial v} \quad & {\left[\left(g_{L}\left(V_{L}-v\right)+g\left(V_{E}-v\right)\right) p_{\varepsilon}\right]+\frac{1}{\varepsilon} \frac{\partial}{\partial g}\left[\left(G_{e q}\left(v, b \mathcal{N}_{\varepsilon}(t)\right)-g\right) p_{\varepsilon}\right] } \\
& -\frac{a}{\varepsilon} \frac{\partial^{2}}{\partial g^{2}} p_{\varepsilon}+\phi_{F}(v) p_{\varepsilon}=0, \quad t \geq 0, V_{R}<v<V_{E}, g \geq 0,
\end{aligned}
$$

with the no-flux boundary conditions

$$
\left\{\begin{array}{l}
\left(G_{e q}\left(v, b \mathcal{N}_{\varepsilon}\right)-g\right) p_{\varepsilon}(v, g, t)-a \frac{\partial}{\partial g} p_{\varepsilon}(v, g, t)=0 \quad \text { for } g=0, \\
{\left[g_{L}\left(V_{L}-V_{R}\right)+g\left(V_{E}-V_{R}\right)\right] p_{\varepsilon}\left(V_{R}, g, t\right)=N_{\varepsilon}(t, g) \quad \text { and } \quad p_{\varepsilon}\left(V_{E}, g, t\right)=0,}
\end{array}\right.
$$

and with an initial data that satisfies

$$
p^{0}(v, g) \geq 0, \quad \int_{V_{R}}^{V_{E}} \int_{0}^{\infty} p^{0}(v, g) d v d g=1 .
$$

A neuron spikes with a rate $\phi_{F}(v)$, typically very large for $v$ larger than a firing potential, and we assume that its membrane potential is instantaneously set at the reset potential $V_{R}$. Therefore, at each time, the firing rate (activity of the network) for neurons with conductance $g$ and respectively the total firing rate of the network are defined as

$$
N_{\varepsilon}(g, t):=\int_{V_{R}}^{V_{E}} \phi_{F}(v) p_{\varepsilon} d v \geq 0, \quad \mathcal{N}_{\varepsilon}(t):=\int_{0}^{+\infty} N_{\varepsilon}(g, t) d g .
$$

Those definitions and boundary conditions, when integrating the equation (1), imply the conservation property

$$
\int_{V_{R}}^{V_{E}} \int_{0}^{\infty} p_{\varepsilon}(v, g, t) d v d g=1
$$

which is in accordance with the interpretation that the solution is the probability density of neurons with potential $v$ and conductance $g$ at time $t$.

For the ease of use, we summarize the parameter interpretation, according to [1, 2],

- $V_{E}$ is the excitatory reversal potential,

- Firing occurs with a rate $\phi_{F}(v) \geq 0, \phi_{F}^{\prime}(v)>0$,

- Reset is at $V_{R}$,

- $V_{L}$ is the leak potential,

- $g_{L}>0$ denotes the leak conductance,

- $G_{e q}(v, \cdot) \geq 0$ is the conductance equilibrium (when ignoring noise),

- $a$ represents the intensity of the synaptic noise,

- $\varepsilon>0$ denotes the time decay constant of the excitatory conductance,

- $b \geq 0$ denotes the synaptic strength of network excitatory coupling.

Concerning parameter range, in [14], see Eq. (3.2a), the authors choose $V_{R}=V_{L}$. Here we consider the more general case $V_{R} \leq V_{L}$. Also, to establish (1), [1,2] assume that the value $\varepsilon$ is small enough. 
Following [1, 14], this motivates to consider the limit $\varepsilon \rightarrow 0$ of Equation (1), which formally leads to a reduction of dimension with a $(1+1)$ dimensional equation easier to tackle. More precisely, we are going to show that it can be described by the following voltage only integrate\&fire model of neural network (see [15, 16, 17, 18, 19] and references therein for the study of PDE Integrate and Fire type models).

$$
\left\{\begin{array}{l}
\frac{\partial}{\partial t} n+\frac{\partial}{\partial v}[\mathcal{G}(v, b \mathcal{N}(t))(V(b \mathcal{N}(t))-v) n]+\phi_{F}(v) n=0, \quad t \geq 0, V_{R}<v<V_{E}, \\
\mathcal{N}(t)=\int_{V_{R}}^{V_{E}} \phi_{F}(v) n(t, v) d v \\
\mathcal{G}\left(V_{R}, b \mathcal{N}(t)\right)\left(V(b \mathcal{N}(t))-V_{R}\right) n\left(t, V_{R}\right)=\mathcal{N}(t) \quad \text { and } \quad n\left(t, V_{E}\right)=0
\end{array}\right.
$$

Our purpose is then, on the one hand, to derive this system in the slow-fast limit $\varepsilon \rightarrow 0$, to explain how noise comes in the expression of the drift, together with the property

$$
V_{L}<V(b \mathcal{N}(t)) \leq V_{E}, \quad \mathcal{G}(v, b \mathcal{N}(t))>0,
$$

which explains the possibility to state the boundary conditions in (6). On the other hand, we aim at studying the qualitative properties of Equation (6), in order to formally obtain, with this more simplified equation, the main properties of Equation (1).

Assumptions on $\phi$ and $G$ and initial data. In the rest of the paper, we make the following assumptions. We assume that the firing rate satisfies

$$
\phi_{F}, \phi_{F}^{\prime} \in L^{\infty}, \quad \phi_{F}^{\prime} \geq 0 .
$$

We also assume that $G(v, A) \geq 0$ and that there is a smooth increasing function $\bar{G} \geq 0$ such that,

$$
\sup _{V_{R} \leq v \leq V_{E}}\left[G(v, A)+\left|\frac{\partial G(v, A)}{\partial v}\right|\right] \leq \bar{G}(A), \quad \forall A \geq 0 .
$$

For example, in $[1,2]$, we find the choice $G(v, b \mathcal{N})=b \mathcal{N}$.

Finally, for the initial data, we assume, for $\varepsilon \leq 1$ and $k \geq 0$

$$
\int_{V_{R}}^{V_{E}} \int_{g=0}^{+\infty} g^{k} p^{0}(v, g) d v d g \leq \mathcal{Q}^{(k), 0}<\infty
$$

The rest of the paper is organized as follows. Section 2 is devoted to the slow-fast limit study of Equation (1) to Equation (6). To this, we preliminary explain how to obtain the formal derivation. We then give uniforms estimates on the moments of the solution and of the firing rate with respect to $\varepsilon$, leading to prove rigorously the weak convergence of Equation (1) to Equation (6) when $\varepsilon$ goes to 0 . We finish this section by the construction of super-solutions leading to uniform $L^{\infty}$ bounds for the solutions of (1) with respect to $\varepsilon$ and for the solutions of Equation (6). In Section 3 we give the main qualitative properties of Equation (6) with a slightly simplified drift. To this, we first study the steady states of this equation as well as the asymptotic long time convergence to those steady states when the strength of connectivity parameter $b$ is small enough. The method of proof is based on entropy methods. We finally consider the case with stronger interconnections where instabilities is proved for the linearized problem and we perform numerical simulations in order to illustrate the emergence of oscillatory solutions. We finish the paper with conclusions and perspectives. 


\section{From conductance-voltage to voltage only model}

Departing with a formal derivation, we first explain, how, Equation (1) leads to Equation (6) when $\varepsilon$ goes to 0 . We then prove uniform estimates on the total firing rate and on the moments on the solution with respect to $\varepsilon$. This leads us to prove rigorously weak convergence of solutions of Equation (1) to Equation (6) (see Theorem 1). Finally, we prove uniform estimates in $L^{\infty}$ when $V_{R}<V_{L}$ by the construction of super-solutions in Theorem 2.

\subsection{Formal derivation}

The relation between the equations (6) and (1) can be simply observed setting

$$
n_{\varepsilon}(v, t)=\int_{0}^{\infty} p_{\varepsilon}(v, g, t) d g
$$

Then, integration in $g$ of (1), using the no-flux boundary condition in $g$, gives

$$
\left\{\begin{array}{l}
\frac{\partial}{\partial t} n_{\varepsilon}+\frac{\partial}{\partial v}\left[\mathcal{G}_{\varepsilon}(v, t)\left(V_{\varepsilon}(t)-v\right) n_{\varepsilon}\right]+\phi_{F}(v) n_{\varepsilon}=0, \quad t \geq 0, V_{R}<v<V_{E} \\
\mathcal{N}_{\varepsilon}(t)=\int_{V_{R}}^{V_{E}} \phi_{F}(v) n_{\varepsilon}(v, t) d v \\
\mathcal{G}_{\varepsilon}\left(V_{R}, t\right)\left(V_{\varepsilon}(t)-V_{R}\right) n_{\varepsilon}\left(t, V_{R}\right)=\mathcal{N}(t) \quad \text { and } \quad n_{\varepsilon}\left(t, V_{E}\right)=0
\end{array}\right.
$$

with the bulk conductance and voltage

$$
\left\{\begin{array}{l}
g_{L} \leq \mathcal{G}_{\varepsilon}(v, t)=\int_{0}^{\infty}\left(g_{L}+g\right) \frac{p_{\varepsilon}(v, g, t)}{n_{\varepsilon}(v, t)} d g \\
V_{L}<V_{\varepsilon}(v, t)=\frac{1}{\mathcal{G}_{\varepsilon}(v, t)} \int_{0}^{\infty}\left(g_{L} V_{L}+g V_{E}\right) \frac{p_{\varepsilon}(v, g, t)}{n_{\varepsilon}(v, t)} d g \leq V_{E}
\end{array}\right.
$$

In order to close this formula, we need to identify the first moment in $g$ of the distribution $p_{\varepsilon}(v, g, t)$ in terms of $n_{\varepsilon}$.

To do so, we consider the limit $p$ of $p_{\varepsilon}$ and it formally solves

$$
\frac{\partial}{\partial g}\left[\left(G_{e q}(v, b \mathcal{N}(t))-g\right) p\right]-a \frac{\partial^{2}}{\partial g^{2}} p=0
$$

With the no-flux boundary condition, we find

$$
\left(G_{e q}(v, b \mathcal{N}(t))-g\right) p-a \frac{\partial p}{\partial g}=0, \quad \text { thefore } \quad p=n(t, v) \mathcal{P}(v, g, b \mathcal{N}(t))
$$

with $n$ solution of Equation (6) and

$$
\mathcal{P}(v, g, A)=\frac{1}{\mathcal{Z}(v, a, A)} \exp ^{-\frac{\left(G_{e q}(v, A)-g\right)^{2}}{2 a}}, \quad \mathcal{Z}(v, a, A)=\int_{0}^{\infty} \exp ^{-\frac{\left(G_{e q}(v, A)-g\right)^{2}}{2 a}} d g
$$

This expression allows us to compute the limiting flux in Equation (6) as stated Theorem 1 below. 
Notice that $\mathcal{Z}(v, a, A)$ is controlled from above and below as

$$
\frac{1}{2} \sqrt{2 \pi a}=\int_{G_{e q}(v, A)}^{\infty} \exp -\frac{\left(G_{e q}(v, A)-g\right)^{2}}{2 a} d g \leq \mathcal{Z}(v, a, A) \leq \sqrt{2 \pi a} .
$$

The effect of noise at the synaptic level is not a diffusion in $v$, as it is assumed in the standard Integrate and Fire model, see for instance [20]. Noise rises a change of the excitatory bulk conductance $\mathcal{G}_{\varepsilon}(v, t)$. It can be compared to the noiseless case $a \rightarrow 0$ which gives

$$
\mathcal{G}_{\varepsilon}\left(v, 0, b \mathcal{N}_{\varepsilon}(t)\right)=G_{e q}\left(v, b \mathcal{N}_{\varepsilon}(t)\right)
$$

In mathematical terms, the analysis of the limit $a \rightarrow 0$, i.e., the case without noise, yields difficulties similar to those of friction dominant particle flows, see [21] for instance.

\subsection{Estimates on the firing rate/moments on the solution and rigorous limit}

We now prove rigorously the above formal derivation. The result is stated in the following Theorem

Theorem 1 (Slow-fast limit result). We assume (8)-(10). Then, for all $k \geq 1$, there exists a constant $C(k)>0$ and a constant $C>0$ such that, for all $\varepsilon>0$ and $t \geq 0$, the following estimates hold

$$
\int_{0}^{+\infty} g^{k} p_{\varepsilon}(v, g, t) d v d g \leq C(k) \quad \text { and } \quad\left\|\mathcal{N}_{\varepsilon}\right\|_{L^{\infty}\left(\mathbb{R}^{+}\right)}+\left\|\mathcal{N}_{\varepsilon}^{\prime}\right\|_{L^{\infty}\left(\mathbb{R}^{+}\right)} \leq C .
$$

Hence, in the weak topology of bounded measures,

$$
p_{\varepsilon} \rightarrow n(t, v) \mathcal{P}(v, g, b \mathcal{N}(t))>0, \quad \int_{0}^{\infty} \mathcal{P}(v, g, b \mathcal{N}(t)) d g=1
$$

where the smooth function $\mathcal{P}(v, g, b \mathcal{N}(t))$ is determined by (13) and where $n(t, v)$ satisfies Equation (6) with the initial data $n^{0}(v)=\int_{0}^{+\infty} p^{0}(v, g) d g$ and

$$
\mathcal{G}(v, A)=g_{L}+\int_{0}^{+\infty} g \mathcal{P}(v, g, A) d g>g_{L}, \quad V_{L}<V(v, A)=\frac{g_{L} V_{L}+V_{E} \int_{0}^{+\infty} g \mathcal{P}(v, g, A) d g}{\mathcal{G}(v, A)}<V_{E}
$$

This theorem states a general weak convergence result. It can be strengthen, with the expense on stronger assumptions as stated afterwards, see Theorem 2 in the next subsection.

\section{Proof of Theorem 1.}

Moments estimates. Let us first prove the first inequality of (14). We set

$$
\mathcal{Q}_{\varepsilon}^{(k)}(t):=\int_{V_{R}}^{V_{E}} \int_{0}^{+\infty} g^{k} p_{\varepsilon}(t, v, g) d v d g .
$$

Multiplying Equation (1) by $g^{k}$ and integrating, we compute for $k \geq 2$

$$
\frac{1}{k} \frac{d}{d t} \mathcal{Q}_{\varepsilon}^{(k)}(t)=\frac{1}{\varepsilon} \int_{V_{R}}^{V_{E}} \int_{0}^{+\infty} g^{k-1}\left[G_{e q}\left(v, b \mathcal{N}_{\varepsilon}(t)\right)-g\right] p_{\varepsilon} d v d g-\frac{a}{\varepsilon} \int_{V_{R}}^{V_{E}} \int_{0}^{+\infty} g^{k-1} \frac{\partial p_{\varepsilon}}{\partial g} d v d g
$$


Therefore we find

$$
\frac{d}{d t} \mathcal{Q}_{\varepsilon}^{(k)}(t)+\frac{k}{\varepsilon} \mathcal{Q}_{\varepsilon}^{(k)}(t)=\frac{k}{\varepsilon} \int_{V_{R}}^{V_{E}} \int_{0}^{+\infty} g^{k-1} G_{e q}\left(v, b \mathcal{N}_{\varepsilon}(t)\right) p_{\varepsilon} d v d g+k(k-1) \frac{a}{\varepsilon} \int_{V_{R}}^{V_{E}} \int_{0}^{+\infty} g^{k-2} p_{\varepsilon} d v d g .
$$

As $p_{\varepsilon}$ is a density probability, then

$$
\left\|\mathcal{N}_{\varepsilon}\right\|_{L^{\infty}}\left(\mathbb{R}^{+}\right) \leq\left\|\phi_{F}\right\|_{L^{\infty}}
$$

Using assumption (9), we deduce that there exists a constant $C$ such that

$$
\int_{V_{R}}^{V_{E}} \int_{0}^{+\infty} g^{k-1} G_{e q}\left(v, b \mathcal{N}_{\varepsilon}(t)\right) p_{\varepsilon} d v d g \leq C \int_{V_{R}}^{V_{E}} \int_{0}^{+\infty} g^{k-1} p_{\varepsilon} d v d g .
$$

Using again that $p_{\varepsilon}$ is a density probability, and splitting the integral in $g$ in two parts : from 0 to $\mu$ and from $\mu$ to $+\infty$, we deduce that for all $\mu>0$ large enough, there exists a constant $C(\mu)$ such that

$$
\int_{V_{R}}^{V_{E}} \int_{0}^{+\infty}\left(g^{k-1}+g^{k-2}\right) p_{\varepsilon} d v d g \leq C(\mu)+\frac{1}{\mu} \mathcal{Q}_{\varepsilon}^{(k)}(t)
$$

Consequently, we have for all $\mu>0$ large enough

$$
\frac{d}{d t} \mathcal{Q}_{\varepsilon}^{(k)}(t)+\frac{k}{\varepsilon} \mathcal{Q}_{\varepsilon}^{(k)}(t) \leq \frac{1}{\varepsilon}\left(C(k, \mu)+\frac{1}{\mu} \mathcal{Q}_{\varepsilon}^{(k)}(t)\right) .
$$

Taking $\mu$ large enough and using Gronwall inequality, we deduce the first part of estimate (14).

Uniform estimates on the firing rate. The bound on $\mathcal{N}_{\varepsilon}(t)$ is easy to obtain. Because the total mass of $p_{\varepsilon}$ is 1 , we conclude from its definition that

$$
\mathcal{N}_{\varepsilon}(t) \leq\left\|\phi_{F}\right\|_{L^{\infty}}
$$

Next, we prove the Lipschitz bound. We multiply equation (1) by $\phi_{F}$ and integrate in $(v, g)$, we find

$$
\begin{aligned}
\left|\frac{d}{d t} \mathcal{N}_{\varepsilon}(t)\right| & \leq\left|\int \phi_{F}^{\prime}(v)\left[g_{L}\left(V_{L}-v\right)+g\left(V_{E}-v\right)\right] p_{\varepsilon}(v, g, t) d g d v\right| \\
& \leq\left\|\phi_{F}^{\prime}(v)\right\|_{L^{\infty}} V_{E} \int g p_{\varepsilon}(v, g, t) d g d v
\end{aligned}
$$

and we conclude using that the moments are uniformly bounded with respect to $\varepsilon$, thanks to (14).

Weak convergence. Let us first deal with the term $G_{e q}\left(v, b \mathcal{N}_{\varepsilon}(t)\right)$. With the second part of estimate (14), we deduce using the Ascoli theorem that, up to a subsequence, there exists a Lipschitz function $\mathcal{N}$ such that for all $T>0$,

$$
\lim _{\varepsilon \rightarrow 0}\left\|\mathcal{N}_{\varepsilon}-\mathcal{N}\right\|_{L^{\infty}(0, T)}=0 .
$$

As $G$ is assumed to be regular, we deduce that, up to a subsequence, for all $T>0$,

$$
\lim _{\varepsilon \rightarrow 0}\left\|G\left(v, b \mathcal{N}_{\varepsilon}\right)-G(v, b \mathcal{N})\right\|_{L^{\infty}\left(\left(V_{R}, V_{E}\right) \times(0, T)\right)}=0 .
$$


Let us now study the convergence of $p_{\varepsilon}$. As $p_{\varepsilon}$ is a density measure and as its moments is uniformly bounded with respect to $\varepsilon$, we deduce that up to a subsequence, there exists a bounded measure function $p$ with total mass 1 and finite moments such that

$$
p_{\varepsilon} \rightarrow p \quad g p_{\varepsilon} \rightarrow g p .
$$

Integrating Equation (1) with respect to the variable $g$, we find that

$$
\widetilde{n}(t, v):=\int_{0}^{+\infty} p(t, v, g) d v
$$

is solution of the equation

$$
\partial_{t} \widetilde{n}(t, v)+\partial_{v}\left(g_{L}\left(V_{L}-v\right) \widetilde{n}+\left(V_{E}-v\right) \int_{0}^{+\infty} g p d g\right)+\phi(v) \widetilde{n}(t, v)=\delta_{v=V_{R}} \mathcal{N}(t)
$$

Combining this with the equality

$$
\frac{\partial}{\partial g}\left[\left(G_{e q}(v, b \mathcal{N}(t))-g\right) p\right]-a \frac{\partial^{2}}{\partial g^{2}} p=0
$$

and following the subsection 2.1, we conclude the proof of Theorem 1 .

\section{$2.3 \quad L^{\infty}$ bound on $p_{\varepsilon}$ and $n$}

We now wish to prove uniform bounds on $p_{\varepsilon}(t, v, g)$ for the full problem (1). For this, we need a kind of non-characteristic condition between the transport in $v$ and the boundary flux at $V_{R}$. Hence, to avoid technicalities, we assume that $V_{R}<V_{L}$ for Equation (1). For the limit Equation (6), the above condition can be relaxed with $V_{R} \leq V_{L}$. The following theorem holds

Theorem 2 ( $L^{\infty}$ bound for $p_{\varepsilon}$ and for $\left.n\right)$. We assume (8)-(10), $V_{R}<V_{L}$, that $p_{\varepsilon}(t=0) \in L^{\infty}$ with sufficient (Gaussian) decay at $g=\infty$ uniformly in $\varepsilon$. Then, for all $T>0$, there exists a constant $C(T)$ independent of $\varepsilon$ small enough such that the solution of (15) satisfies

$$
\begin{gathered}
\sup _{0 \leq t \leq T} \sup _{g \geq 0} N_{\varepsilon}(t, g) \leq C(T), \\
\sup _{0 \leq t \leq T} \sup _{V_{R} \leq v \leq V_{E}} \sup _{g \geq 0} p_{\varepsilon}(t, v, g) \leq C(T) .
\end{gathered}
$$

Assume that $V_{R} \leq V_{L}$ and (8)-(10). Then, there is a constant $C(T)$ such that, the solutions of (6) satisfy

$$
\sup _{0 \leq t \leq T} \sup _{V_{R} \leq v \leq V_{E}} n(t, v) \leq C(T)\left\|n^{0}\right\|_{L^{\infty}}
$$

Proof of Theorem 2. We begin with the estimates on $p_{\varepsilon}$ and then treat those for $n$. 
Estimate for $p_{\varepsilon}$. We consider $\mathcal{N}_{\varepsilon}(t)$ as a given data in the term $G_{e q}$ and we build a super solution $\bar{p}_{\varepsilon}(v, g, t)$ of Equation (1) for $p_{\varepsilon}(v, g, t)$, that is a solution of the following Problem for $t \geq 0, g \geq 0$ and $V_{R}<v<V_{E}$,

$$
\left\{\begin{array}{l}
\frac{\partial}{\partial t} \bar{p}_{\varepsilon}+\frac{\partial}{\partial v}\left[\left(g_{L}\left(V_{L}-v\right)+g\left(V_{E}-v\right)\right) \bar{p}_{\varepsilon}\right]+\frac{1}{\varepsilon} \frac{\partial}{\partial g}\left[\left(G_{e q}\left(v, b \mathcal{N}_{\varepsilon}(t)\right)-g\right) \bar{p}_{\varepsilon}\right]-\frac{a}{\varepsilon} \frac{\partial^{2}}{\partial g^{2}} \bar{p}_{\varepsilon} \geq 0 \\
a \frac{\partial}{\partial g} \bar{p}_{\varepsilon}-\left(G_{e q}\left(v, b \mathcal{N}_{\varepsilon}(t)\right)-g\right) \bar{p}_{\varepsilon}=0, \quad \text { at } g=0, \quad p_{\varepsilon}\left(V_{E}, g, t\right)=0 \\
{\left[g_{L}\left(V_{L}-V_{R}\right)+g\left(V_{E}-V_{R}\right)\right] \bar{p}_{\varepsilon}\left(V_{R}, g, t\right) \geq N_{\varepsilon}(g, t):=\int \phi_{F}(v) p_{\varepsilon}(v, g, t) d v .}
\end{array}\right.
$$

We construct it under the form

$$
\bar{p}_{\varepsilon}(t, v, g)=B e^{\alpha\left(V_{R}-v\right)} M(v, g, t) e^{\varepsilon g^{2}} e^{\mu t}, \quad \text { with } M(t, v, g)=\exp ^{-\frac{\left(G_{e q}\left(v, b \mathcal{N}_{\varepsilon}(t)\right)-g\right)^{2}}{2 a}},
$$

with $B, \alpha$ and $\mu$ three constants which are large enough.

Firstly, the constant $B$ is just used to satisfy the initial condition and we ignore it in the end of the proof.

Secondly, we fix $\alpha$ so that the boundary condition at $V_{L}$ is fulfilled. That simply means (recall that we assume $V_{R}<V_{L}$ )

$$
\left[g_{L}\left(V_{L}-V_{R}\right)+g\left(V_{E}-V_{R}\right)\right] M\left(t, V_{R}, g\right) \geq \int \phi_{F}(v) e^{\alpha\left(V_{R}-v\right)} M(t, v, g) d v
$$

which is possible, because large values of $g$ are favorable, for $\alpha$ large enough.

Thirdly, because $M(t, v, g)$ satisfies

$$
\left(G_{e q}-g\right) M-a \partial_{g} M=0
$$

we deduce that

$$
a \partial_{g} \bar{p}_{\varepsilon}-\left(G_{e q}-g\right) \bar{p}_{\varepsilon}=2 a \bar{p}_{\varepsilon} \varepsilon g \geq 0,
$$

and hence, the zero flux boundary condition is satisfied at $g=0$.

Finally, building on the above calculation, we also find successively that

$$
\begin{gathered}
\partial_{g}\left(\left(G_{e q}-g\right) \bar{p}_{\varepsilon}-a \partial_{g} \bar{p}_{\varepsilon}\right)=-2 a \varepsilon B e^{\mu t} \partial_{g}\left(g M_{\varepsilon} e^{\varepsilon g^{2}}\right), \\
\partial_{g}\left(\left(G_{e q}-g\right) \bar{p}_{\varepsilon}-a \partial_{g} \bar{p}_{\varepsilon}\right)=\left(-2 a \varepsilon-a(2 g \varepsilon)^{2}+\varepsilon g\left(g-G_{e q}\right)\right) \bar{p}_{\varepsilon} .
\end{gathered}
$$

We deduce that there exists a constant $C$ independent of $\varepsilon$ small enough, such that for all $v \in\left(V_{R}, V_{E}\right)$ for all $g \geq 0$

$$
\varepsilon^{-1} \partial_{g}\left(\left(G_{e q}-g\right) \bar{p}_{\varepsilon}-a \partial_{g} \bar{p}_{\varepsilon}\right) \geq\left[\frac{g^{2}}{2}-C(1+g)\right] \bar{p}_{\varepsilon} .
$$

On the other hand, we have

$$
\partial_{t} \bar{p}_{\varepsilon}=\left(\mu+z_{\varepsilon}(t, v, g)\right) \bar{p}_{\varepsilon}
$$


with

$$
z_{\varepsilon}(t, v, g)=\frac{b}{a} \frac{\partial G_{e q}}{\partial A}\left(g-G_{e q}\right) \frac{d}{d t} \mathcal{N}_{\varepsilon}(t) .
$$

Using that $\mathcal{N}_{\varepsilon}$ is uniformly Lipschitz with respect to $\varepsilon$, see (14), we deduce that there exists a constant $C$ independent of $\varepsilon$ such that for all $v \in\left(V_{R}, V_{E}\right)$ for all $g \geq 0$

$$
\left|z_{\varepsilon}\right| \leq C(1+g) \text {. }
$$

We then deduce that

$$
\partial_{t} \bar{p}_{\varepsilon} \geq(\mu-C(1+g)) \bar{p}_{\varepsilon} .
$$

Simple computations show that there exists a constant $C$ independent of $\varepsilon$ such that for all $v \in\left(V_{R}, V_{E}\right)$ for all $g \geq 0$

$$
\partial_{v}\left(\left(g_{L}\left(V_{L}-v\right)+g\left(V_{E}-v\right)\right) \bar{p}_{\varepsilon}\right) \geq-C(1+\alpha)(1+g) \bar{p}_{\varepsilon} .
$$

Combining (16), (17), (18) and taking $\mu$ large enough, we deduce that

$$
\partial_{t} \bar{p}_{\varepsilon}+\partial_{v}\left[\left(g_{L}\left(V_{L}-v\right)+g\left(V_{E}-v\right)\right) \bar{p}_{\varepsilon}\right]+\frac{1}{\varepsilon} \partial_{g}\left[\left(G_{e q}-g\right) \bar{p}_{\varepsilon}-a \partial_{g} \bar{p}_{\varepsilon}\right] \geq 0 .
$$

This proves the first part of Theorem 2 .

Estimate for $n$. Due to (7), there exists a constant $\underline{b}>0$, such that

$$
\mathcal{G}\left(V_{R}, A\right)\left(V\left(V_{R}, A\right)-V_{R}\right) \geq \underline{b}>0 .
$$

Moreover, as $n$ is a probability density, we have

$$
\|\mathcal{N}\|_{L^{\infty}} \leq\left\|\phi_{F}\right\|_{L^{\infty}} .
$$

To show that we can build a super-solution, we can reduced to construct $\bar{n} \geq 0$ such that

$$
\partial_{t} \bar{n}+\partial_{v}[\mathcal{G}(v, b \mathcal{N}(t))(V(b \mathcal{N}(t))-v) \bar{n}] \geq 0
$$

and

$$
\left(\mathcal{G}\left(V_{R}, b \mathcal{N}(t)\right)\left(V(b \mathcal{N}(t))-V_{R}\right) \bar{n}\right) \geq\left\|\phi_{F}\right\|_{L^{\infty}} .
$$

Choosing

$$
\bar{n}(t, v):=B\left\|n^{0}\right\|_{L^{\infty}} e^{A t}
$$

with $A$ and $B$ large enough and using assumption (19), we deduce that $\bar{n}$ is a super-solution which ends the proof of Theorem 2 .

\section{Qualitative study of the associated Integrate and Fire model}

This section is devoted to the understanding of the qualitative and asymptotic behavior of Equation (6) with respect to the strength of interconnections of the network. To simplify the presentation, we consider a slightly simplified model of Equation (6) as follows.

$$
\left\{\begin{array}{l}
\frac{\partial n}{\partial t}+\frac{\partial}{\partial v}\left[\left(V_{0}(\mathcal{N})-v\right) n\right]+\phi_{F}(v) n=0, \quad 0 \leq v \leq V_{E} \\
V_{0}(\mathcal{N}(t)) n(t, v=0)=\mathcal{N}, \quad n\left(t, V_{E}\right)=0, \quad \mathcal{N}(t)=\int_{0}^{V_{E}} \phi_{F}(v) n(t, v) d v .
\end{array}\right.
$$

Here, firstly, following [1, 2], we choose $V_{L}=V_{R}=0$. Secondly, we use a simpler drift $V_{0}$ independent of the variable $v$. 


\subsection{Steady states}

The associated steady equation is given by

$$
\left\{\begin{array}{l}
\frac{\partial}{\partial v}\left[\left(V_{0}(\overline{\mathcal{N}})-v\right) \bar{n}\right]+\phi_{F}(v) \bar{n}=0, \quad 0 \leq v \leq V_{E} \\
V_{0}(\overline{\mathcal{N}}) \bar{n}(v=0)=\overline{\mathcal{N}}, \quad p\left(V_{E}\right)=0, \quad \overline{\mathcal{N}}=\int_{0}^{V_{E}} \phi_{F}(v) \bar{n}(v) d v .
\end{array}\right.
$$

We assume that the smooth function $V_{0}(\cdot)$ satisfies

$$
0<V_{0}(\mathcal{N})<V_{E}, \quad \forall \mathcal{N} \geq 0
$$

One observes that, to avoid concentration as a Dirac mass at $v=V_{0}(\mathcal{N})$, it is useful to also assume

$$
\phi_{F}\left(V_{0}(\mathcal{N})\right)>0 \quad \forall \mathcal{N} \geq 0 .
$$

Indeed, we may write Equation (21) as

$$
\left(V_{0}(\overline{\mathcal{N}})-v\right) \frac{\partial \bar{n}}{\partial v}+\left(\phi_{F}(v)-1\right) \bar{n}=0 .
$$

Therefore, near $v=V_{0}(\overline{\mathcal{N}})$, the solution behaves as $\frac{\partial \ln (\bar{n})}{\partial v}=-\frac{\phi_{F}\left(V_{0}(\overline{\mathcal{N}})\right)-1}{V_{0}(\overline{\mathcal{N}})-v}$ and thus, for some constant $q_{s}$

$$
\bar{n} \approx q_{s}\left(V_{0}(\overline{\mathcal{N}})-v\right)^{\alpha}, \quad \alpha=\phi_{F}\left(V_{0}(\overline{\mathcal{N}})\right)-1>-1,
$$

which means that Condition (23) implies that $\bar{n}$ has an integrable singularity.

More precisely we have

Theorem 3. With the assumptions (8), (22), (23), there is at least one steady state solution $\bar{n}$ which satisfies $\int_{0}^{V_{E}} \bar{n}=1$.

Proof. It is possible to give an expression of the stationary solution $\bar{n}$. Using (24), we can write

$$
\frac{\partial \ln (\bar{n})}{\partial v}= \begin{cases}\frac{1-\phi_{F}(v)}{V_{0}(\overline{\mathcal{N}})-v}, & \text { for } v<V_{0}(\overline{\mathcal{N}}) \\ 0, & \text { for } v>V_{0}(\overline{\mathcal{N}})\end{cases}
$$

Therefore we define

$$
F(v, \overline{\mathcal{N}})=\int_{0}^{v} \frac{1-\phi_{F}(w)}{V_{0}(\overline{\mathcal{N}})-w} d w
$$

and we conclude that (and recall this $\bar{n}$ is integrable near $V_{0}(\overline{\mathcal{N}})$ as we saw it above)

$$
\bar{n}(v)= \begin{cases}\frac{\overline{\mathcal{N}}}{V_{0}(\overline{\mathcal{N}})} e^{F(v, \overline{\mathcal{N}})}, & \text { for } v<V_{0}(\overline{\mathcal{N}}), \\ 0, & \text { for } v>V_{0}(\overline{\mathcal{N}}) .\end{cases}
$$

Indeed, from (24), we may also infer that $\bar{n} \equiv 0$ for $v>V_{0}(\overline{\mathcal{N}})$ and the sign of $\frac{\partial \bar{n}}{\partial v}$ is the sign of $1-\phi_{F}(v)$. Next, we choose the value $\overline{\mathcal{N}}$ so as to enforce the constraint $\int_{0}^{V_{0}(\overline{\mathcal{N}})} \bar{n}=1$. For $\overline{\mathcal{N}}=0$, the corresponding solution $\bar{n}$ vanishes, and for $\overline{\mathcal{N}} \rightarrow \infty$, we have $\bar{n} \rightarrow \infty$. By continuity, we may achieve the constraint.

These considerations explain the numerical solutions depicted in the Figure 1. These are obtained with

$$
V_{R}=0, \quad V_{E}=1, \quad \phi_{F}(v)=A \mathbb{I}_{\{v>.5\}}, \quad V_{0}(\mathcal{N})=.8+.2 \frac{\mathcal{N}}{1+\mathcal{N}},
$$

and $A=.5$ for the figure on the left, $A=5$. for the figure on the right. The value $V_{0}(\mathcal{N})$ can be identified because the solution vanishes for $v>V_{0}(\mathcal{N})$. 

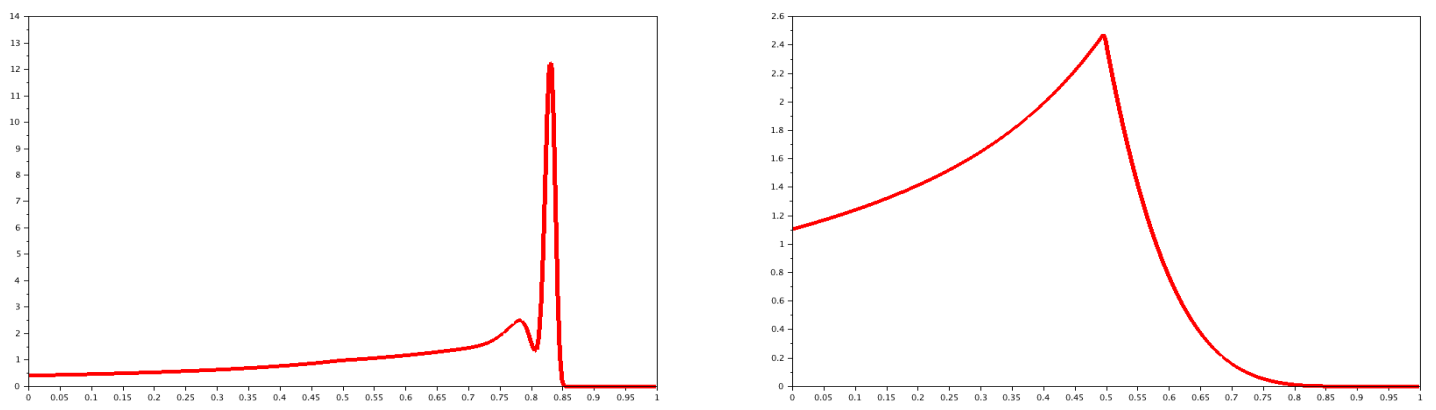

Figure 1: Numerical solutions of the steady states in (21) With the date in (26) And two Choices of the term $\phi_{F}(v)$. Abscissae are $v$. Left: firing rate $A=.5$; Right: Firing rate $A=5$.

Cases of uniqueness of the steady state. We may complete the existence result in Theorem 3 with uniqueness cases

Theorem 4. With the assumptions (22), (23), the steady state is unique in the two following cases. 1. $\phi_{F}>1$ on $\left[0, V_{E}\right]$ and

$$
0<\mathcal{N} V_{0}^{\prime}(\mathcal{N})<V_{0}(\mathcal{N}), \quad \forall \mathcal{N}>0
$$

2. $\phi_{F}^{\prime} \geq 0$ and $V_{0}^{\prime}<0$

Notice that Condition (27) is satisfied, for instance, by $V_{0}(\mathcal{N})=\frac{a \mathcal{N}}{b+\mathcal{N}}+c$, with $a \geq 0, b>0$ and $c \geq 0$, three constants.

\section{Proof of Theorem 4.}

Case 1. The condition providing the steady states in Theorem 3 , that is $\int_{0}^{V_{0}(\overline{\mathcal{N}})} \bar{n}=1$, is also written

$$
\frac{V_{0}(\mathcal{N})}{\mathcal{N}}=\int_{0}^{V_{0}(\mathcal{N})} e^{F(v, \mathcal{N})} d v
$$

and we show that the two uniqueness cases correspond to left and right hand sides with opposite monotonicity.

The derivative of the left hand side is given by

$$
\frac{\mathcal{N} V_{0}^{\prime}(\mathcal{N})-V_{0}(\mathcal{N})}{\mathcal{N}^{2}}<0
$$

To treat the right hand side, we observe that, thanks to the assumption $\phi_{F}(v)>1$, we have $e^{F\left(\mathcal{N}, V_{R}\right)}=$ 0 . Therefore its sign is given by that of $V_{0}^{\prime}(\mathcal{N})$ because

$$
\frac{\partial F(v, \mathcal{N})}{\partial \mathcal{N}}=-V_{0}^{\prime}(\mathcal{N}) \int_{0}^{v} \frac{1-\phi_{F}(w)}{\left(V_{0}(\mathcal{N})-w\right)^{2}} d w
$$

Because the latter expression has the sign opposite to $V_{0}^{\prime}(\mathcal{N})$ we find the result.

Case 2. To consider also possible values $\phi_{F}<1$ and to compute the derivative in $\mathcal{N}$ requires additional steps. We write, for $v<V_{0}(\mathcal{N})$,

$$
F(v, \mathcal{N})=\int_{0}^{v / V_{0}(\mathcal{N})} \frac{1-\phi_{F}\left(z V_{0}(\mathcal{N})\right)}{1-z} d z, \quad \text { and } \quad G(w, \mathcal{N})=\int_{0}^{w} \frac{1-\phi_{F}\left(z V_{0}(\mathcal{N})\right)}{1-z} d z
$$


The formula (25) gives,

$$
\int_{0}^{V_{0}(\overline{\mathcal{N}})} \bar{n}(v)=\overline{\mathcal{N}} \int_{0}^{1} e^{G\left(w, V_{0}(\overline{\mathcal{N}})\right)} d w
$$

and $\bar{n}$ is a probability measure if

$$
\frac{1}{\overline{\mathcal{N}}}=\int_{0}^{1} e^{G\left(w, V_{0}(\overline{\mathcal{N}})\right)} d w
$$

Uniqueness follows under the condition that $G$ is increasing in $\mathcal{N}$, which means

$$
-\phi_{F}^{\prime}\left(z V_{0}(\mathcal{N})\right) V_{0}^{\prime}(\mathcal{N})>0
$$

and, since $\phi_{F}^{\prime} \geq 0$, this gives the condition $V_{0}^{\prime}<0$.

\section{$3.2 \quad$ Asymptotic stability}

Back to the evolution equation (20), the next step is to determine if the solutions converge to a steady state in long time. We first consider the linear case and by a perturbation method conclude that the result still holds, assuming a weak nonlinearity. We use the Doeblin method which is very well adapted to the problem at hand, see [22, 23, 24] for recent presentations and results.

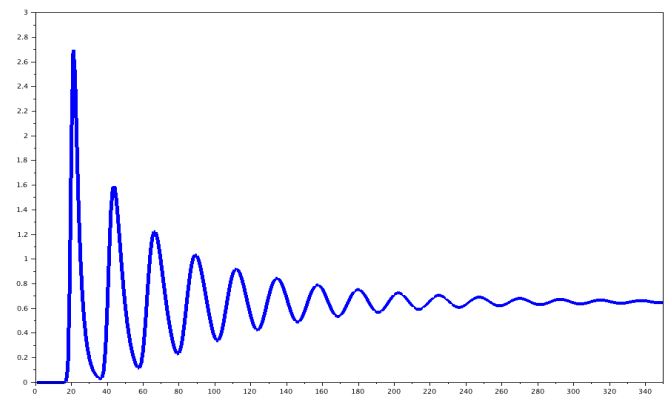

Figure 2: Numerical solutions of the EVolution Equation $(20) V_{0}(\mathcal{N})=.95$, that is the Linear EQUATion. This SHOWs THAT THE SOLUTION RELAXES TO THE STEADY STATE DEPICTED IN Fig. 1 WiTH DAMPED OSCILLATIONS.

Linear case. In the linear case ( $V_{0}$ constant), we may apply the Theorem 2.3 in [22] to equation (20). We obtain the following criteria for exponential convergence to a unique stationary state. If, for a time $t_{0}>0$, we are able to construct non-negative sub-solution, uniformly with respect to all initial data in $L^{1}$, then the solution of our equation converges exponentially to a unique stationary state. More precisely, the following result holds

Theorem 5 ([22]). Assume that $V_{0}>0$ is constant and assume that there exist $t_{0}>0$ and a nonnegtive function $\nu \neq 0$ such that for all initial data $n^{0} \in L_{+}^{1}\left(0, V_{E}\right)$ with $\int_{0}^{V_{E}} n^{0}(v) d v=1$, the solution of $(20)$ at time $t_{0}$ satisfies

$$
n\left(t_{0}, v\right) \geq \nu(v) .
$$

Then, there exists a unique stationary state $\bar{n}$ of Equation (20), there exist $\alpha>0, C>0$ such that for all $t \geq 0$ and for all density initial data $n^{0} \in L^{1}$

$$
\|n(t)-\bar{n}\|_{L^{1}} \leq C e^{-\alpha t}\left\|n^{0}-\bar{n}\right\|_{L^{1}}
$$


Notice that Doeblin's method is particular adapted to work with measures. However, in our context, we have control in $L^{1}$ and thus we restrict ourselves to this context. Once adapted to our case, we conclude that the following result holds

Theorem 6. Assume that $V_{0}$ is constant, $V_{0}>0$ and $\phi_{F}\left(V_{0}\right)>0$. Then, there exists $t_{0}>0$ and a non-negative function $\nu \neq 0$ such that for all initial data $n^{0} \in L_{+}^{1}\left(0, V_{E}\right)$ with $\int_{0}^{V_{E}} n^{0}(v) d v=1$, the solution of (20) at time $t_{0}$ satisfies estimate (28).

As a consequence, there exist $\alpha>0, C>0$ such that for all $t \geq 0$

$$
\|n(t)-\bar{n}\|_{L^{1}} \leq C e^{-\alpha t}\left\|n^{0}-\bar{n}\right\|_{L^{1}},
$$

where $\bar{n}$ is the stationary state of Equation (20).

Proof of Theorem 6. Equation (20) can be written as

$$
\frac{\partial n}{\partial t}+\left(V_{0}-v\right) \frac{\partial n}{\partial v}+\left(\phi_{F}(v)-1\right) n=0
$$

With the method of characteristics, we obtain that

$$
n(t, v)=0 \quad \text { for } \quad v \geq\left[V_{E}-V_{0}\right] e^{-t}+V_{0} .
$$

In the interval

$$
V_{0}\left(1-e^{-t}\right) \leq v \leq\left[V_{E}-V_{0}\right] e^{-t}+V_{0},
$$

we have

$$
n(t, v)=e^{t-\int_{0}^{t} \phi_{F}\left(v+V_{0}\left(e^{-t}-1\right)+V_{0}\left(1-e^{-s}\right)\right) d s} n\left(0, v e^{t}+V_{0}\left(1-e^{t}\right)\right),
$$

and finally, in the interval

$$
0 \leq v \leq V_{0}\left(1-e^{-t}\right)
$$

we have

$$
n(t, v)=\frac{1}{V_{0}} \mathcal{N}\left(t+\ln \left(V_{0}-v\right)-\ln \left(V_{0}\right)\right) e^{-\ln \left(V_{0}-v\right)+\ln \left(V_{0}\right)-\int_{0}^{-\ln \left(V_{0}-v\right)+\ln \left(V_{0}\right)} \phi_{F}\left(V_{0}\left(1-e^{-s}\right)\right) d s .}
$$

Using formula (29), we deduce that

$$
\mathcal{N}(t) \geq e^{t\left(1-\left\|\phi_{F}\right\|_{L} \infty\right)} \int_{V_{0}\left(1-e^{-t}\right)}^{\left[V_{E}-V_{0}\right] e^{-t}+V_{0}} \phi_{F}(v) n^{0}\left(v e^{t}+V_{0}\left(1-e^{t}\right)\right) d v .
$$

Now, we use that $\phi_{F}\left(V_{0}\right)>0$ and that $\phi_{F}$ is regular. This implies that there exist a constant $C>0$ and $t_{1}>0$ such that for

$$
V_{0}\left(1-e^{-t_{1}}\right) \leq v \leq\left[V_{E}-V_{0}\right] e^{-t_{1}}+V_{0}, \quad \text { we have } \phi_{F}(v) \geq C>0 .
$$

Hence, for $t \geq t_{1}$, we have

$$
\mathcal{N}(t) \geq C e^{t\left(1-\left\|\phi_{F}\right\|_{L^{\infty}}\right)} \int_{V_{0}\left(1-e^{-t}\right)}^{\left[V_{E}-V_{0}\right] e^{-t}+V_{0}} n^{0}\left(v e^{t}+V_{0}\left(1-e^{t}\right)\right) d v
$$

Now, using that $\int_{0}^{V_{E}} n^{0}(v) d v=1$, we obtain the following estimate, independent of the initial data, that is

$$
\mathcal{N}(t) \geq C e^{-\left\|\phi_{F}\right\|_{L} \infty t} \quad \forall t \geq t_{1}
$$


Using now formula (30), we deduce that for $t_{0}=2 t_{1}$, and for

$$
v \in\left[0, V_{0}\left(1-e^{-\frac{t_{0}}{2}}\right)\right] \text { with } 0 \leq-\ln \left(V_{0}-v\right)+\ln \left(V_{0}\right) \leq \frac{t_{0}}{2},
$$

we have

$$
n\left(t_{0}, v\right) \geq C e^{-\left\|\phi_{F}\right\|_{L} \infty t_{0}} .
$$

This implies, that there exists a time $t_{0}:=2 t_{1}$ and a nonnegative function $\nu$ with $\int \nu>0$, such that, for all initial data in $L^{1}$, (28) condition is satisfied. This ends the proof of Theorem 6.

Nonlinear case. We now consider the nonlinear Equation (20), which means that $V_{0}$ is not necessary a constant. The following theorem holds

Theorem 7. Assume (22), (23) and that there is a constant D $>0$, small enough, such that

$$
\left(\left\|\left(\frac{N}{V_{0}(N)}\right)^{\prime}\right\|_{L^{\infty}}+\left\|V_{0}^{\prime}\right\|_{L^{\infty}}\right)\left\|\phi_{F}\right\|_{L^{\infty}} \leq D .
$$

Then, there exists a unique stationary state $n^{*}$ of Equation (20) and there exists constants $\alpha>0$ and $C>0$ such that for all initial data $n^{0} \in L^{1}\left(0, V_{E}\right), \int_{0}^{V_{E}} n^{0}(v) d v=1$, the solution of Equation (20) satisfies

$$
\left\|n(t)-n^{*}\right\|_{L^{1}} \leq C e^{-\alpha t}\left\|n 0-n^{*}\right\|_{L^{1}} .
$$

Proof of Theorem 7. As the assumptions of Theorem 3 hold, there exists a stationary state of Equation (20), we denote it by $\left(n^{*}, \mathcal{N}^{*}\right)$. For a density $n^{0}$ as above, we call $S_{t}\left(n^{0}\right)$ the solution of the linear equation

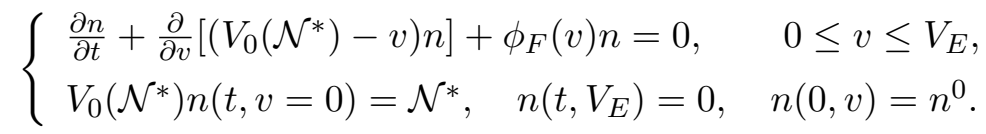

Then, using Duhamel's principle, the solution of Equation (20) with initial data $n^{0}$ can be written as

$$
n(t, v)=S_{t}\left(n^{0}\right)+\int_{0}^{t} S_{t-\tau}\left(V_{0}\left(\mathcal{N}^{*}\right)-V_{0}(\mathcal{N}(\tau)) n(\tau, v) d \tau+\delta_{v=0} \int_{0}^{t} S_{t-\tau}\left(\frac{\mathcal{N}(\tau)}{V_{0}(\mathcal{N}(\tau))}-\frac{\mathcal{N}^{*}}{V_{0}\left(\mathcal{N}^{*}\right)}\right) d \tau .\right.
$$

From this, inserting absolute values, integrating in $v$ and using that the total mass of $n$ is 1 , we obtain

$$
\left\|n(t)-n^{*}\right\|_{L^{1}} \leq\left\|S_{t}\left(n^{0}\right)-n^{*}\right\|_{L^{1}}+\int_{0}^{t}\left|S_{t-\tau}\left(V_{0}\left(\mathcal{N}^{*}\right)-V_{0}(\mathcal{N}(\tau))+\frac{\mathcal{N}(\tau)}{V_{0}(\mathcal{N}(\tau))}-\frac{\mathcal{N}^{*}}{V_{0}\left(\mathcal{N}^{*}\right)}\right)\right| d \tau .
$$

We now use Theorem 6 to obtain two constants $C>0$ and $\beta>0$ such that

$$
\left\|n(t)-n^{*}\right\|_{L^{1}} \leq e^{-\beta t}\left\|n^{0}-n^{*}\right\|_{L^{1}}+\int_{0}^{t} e^{-\beta(t-\tau)}\left|\left(V_{0}\left(\mathcal{N}^{*}\right)-V_{0}(\mathcal{N}(\tau))+\frac{\mathcal{N}(\tau)}{V_{0}(\mathcal{N}(\tau))}-\frac{\mathcal{N}^{*}}{V_{0}\left(\mathcal{N}^{*}\right)}\right)\right| d \tau
$$

To conclude, it remains to estimate

$$
\left|\left(V_{0}\left(\mathcal{N}^{*}\right)-V_{0}(\mathcal{N}(\tau))+\frac{V_{0}(\mathcal{N}(\tau))}{\mathcal{N}(\tau)}-\frac{V_{0}\left(\mathcal{N}^{*}\right)}{\mathcal{N}^{*}}\right)\right| .
$$

To do so, we notice that

$$
\mid V_{0}\left(\mathcal{N}^{*}\right)-V_{0}\left(\mathcal{N}(\tau)\left|\leq\left\|V_{0}^{\prime}\right\|_{L^{\infty}}\right| \mathcal{N}^{*}-\mathcal{N}(\tau) \mid \leq\left\|V_{0}^{\prime}\right\|_{L^{\infty}}\left\|\phi_{F}\right\|_{L^{\infty}}\left\|n(\tau)-n^{*}\right\|_{L^{1}} .\right.
$$


and

$$
\left|\frac{\mathcal{N}(\tau)}{V_{0}(\mathcal{N}(\tau))}-\frac{\mathcal{N}^{*}}{V_{0}\left(\mathcal{N}^{*}\right)}\right| \leq\left\|\left(\frac{N}{V_{0}(N)}\right)^{\prime}\right\|_{L^{\infty}}\left\|\phi_{F}\right\|_{L^{\infty}}\left\|n(\tau)-n^{*}\right\|_{L^{1}} .
$$

With the weak nonlinearity assumption

$$
\mid V_{0}^{\prime}\left\|_{L^{\infty}}\right\| \phi_{F}\left\|_{L^{\infty}}+\right\|\left(\frac{N}{V_{0}(N)}\right)^{\prime}\left\|_{L^{\infty}}\right\| \phi_{F} \|_{L^{\infty}}<\beta,
$$

we conclude Theorem 7 using the Gronwall lemma and choosing

$$
\alpha=\beta-\left\|V_{0}^{\prime}\right\|_{L^{\infty}}\left\|\phi_{F}\right\|_{L^{\infty}}+\left\|\left(\frac{N}{V_{0}(N)}\right)^{\prime}\right\|_{L^{\infty}}\left\|\phi_{F}\right\|_{L^{\infty}} .
$$

\subsection{Oscillatory states}

As observed in [5], the voltage-conductance model (1) can produce periodic solutions which represent the spontaneous activity of the network. This desirable property also holds for other neural network models; see [25] for the Leaky Integrate\&Fire model and [11, 12] for the time elapsed model.

Linear instability. While in Subsections 3.2 and ??, we have studied the nonlinear asymptotic stability of the steady state, one can also find conditions for its instability.

The linearization of Equation (21) around a steady state $\bar{n}(v)$ is to find $(r(v), \mu)$ satisfying

$$
\left\{\begin{array}{l}
\frac{\partial r}{\partial t}+\frac{\partial}{\partial v}\left[\left(\bar{V}_{0}-v\right) r\right]+\bar{V}_{0}^{\prime} \mu \frac{\partial \bar{n}(v)}{\partial v}+\phi_{F} r=0, \quad 0 \leq v \leq V_{E}, \\
\mu:=\int_{0}^{V_{E}} \phi_{F} r d v, \quad \int_{0}^{V_{E}} r d v=0, \quad \bar{V}_{0} r(0)=\mu\left[1-\bar{V}_{0}^{\prime} \bar{n}(0)\right],
\end{array}\right.
$$

with $\bar{V}_{0}=V_{0}(\overline{\mathcal{N}})$.

We look for a solution with exponential behavior in time $r(v) e^{\lambda t}$ which gives

$$
\left\{\begin{array}{l}
\lambda r+\frac{\partial}{\partial v}\left[\left(\bar{V}_{0}-v\right) r\right]+\bar{V}_{0}^{\prime} \mu \frac{\partial \bar{n}(v)}{\partial v}+\phi_{F} r=0, \quad 0 \leq v \leq V_{E} \\
\mu:=\int_{0}^{V_{E}} \phi_{F} r d v, \quad \bar{V}_{0} r(0)=\mu\left[1-\bar{V}_{0}^{\prime} \bar{n}(0)\right] .
\end{array}\right.
$$

Because $\bar{n}(v)=0$ for $v>\bar{V}_{0}$, we also have $r(v)=0$ for $v>\bar{V}_{0}$ and it remains to solve the problem for $v \in\left(0, \bar{V}_{0}\right)$. Notice that the condition $\int_{0}^{V_{E}} r d v=0$ follows by integration when $\lambda \neq 0$.

Proposition 1. We assume that $\phi_{F}(v)=\phi_{1} \mathbb{I}_{\{v>v 1\}}$ and $\phi_{1}>1$. We also assume that

$$
\frac{V_{0}}{\overline{\mathcal{N}}_{0}}-\phi_{1} \bar{V}_{0}^{\prime}+\bar{V}_{0}^{\prime} \frac{\overline{\mathcal{N}}}{\bar{V}_{0}}\left(\bar{V}_{0}-v_{1}\right)^{\phi_{1}-2}<0
$$

Then there is a solution of the problem (33) with $\lambda>0$. In other words the steady state $\bar{n}$ is unstable. 
To solve this problem, we notice that $q=\frac{r}{\bar{n}}$ satisfies

$$
\lambda q+\left(\bar{V}_{0}-v\right) \frac{\partial q}{\partial v}+\frac{\bar{V}_{0}^{\prime} \mu}{\bar{n}} \frac{\partial \bar{n}(v)}{\partial v}=0
$$

and thus, using (21),

$$
\begin{gathered}
\frac{\partial\left(\bar{V}_{0}-v\right)^{\lambda} q}{\partial v}=-\left(\bar{V}_{0}-v\right)^{\lambda-1} \frac{\bar{V}_{0}^{\prime} \mu}{\bar{n}} \frac{\partial \bar{n}(v)}{\partial v}=\bar{V}_{0}^{\prime} \mu\left(\bar{V}_{0}-v\right)^{\lambda-2}\left(\phi_{F}-1\right), \\
\left(\bar{V}_{0}-v\right)^{\lambda} q=\left(\bar{V}_{0}\right)^{\lambda} q(0)+\bar{V}_{0}^{\prime} \mu \frac{\bar{V}_{0}^{\lambda-1}-\left(\bar{V}_{0}-v\right)^{\lambda-1}}{\lambda-1}\left(\left\langle\phi_{F}(v)\right\rangle-1\right)
\end{gathered}
$$

where $\left\langle\phi_{F}(v)\right\rangle$ ranges for some average of $\phi_{F}$ on $(0, v)$.

At this stage we recall the singularity of $\bar{n}(v) \approx q_{s}\left(V_{0}(\overline{\mathcal{N}})-v\right)^{\alpha}, \alpha=\phi_{F}\left(\bar{V}_{0}\right)-1>-1$. This means that $r=q \bar{n}$ is integrable for $\lambda<\phi_{F}\left(\bar{V}_{0}\right), 1<\phi_{F}\left(\bar{V}_{0}\right)$.

Then, eliminating $\mu$, the parameter $\lambda$ is chosen so as to satisfy

$$
1=\left(\bar{V}_{0}\right)^{\lambda-1}\left[\frac{V_{0}}{\overline{\mathcal{N}}_{0}}-\bar{V}_{0}^{\prime}\right] \int \frac{\bar{n}(v)}{\left(\bar{V}_{0}-v\right)^{\lambda}} d v+\bar{V}_{0}^{\prime} \int \frac{\bar{n}(v)}{\left(\bar{V}_{0}-v\right)^{\lambda}} \frac{\bar{V}_{0}^{\lambda-1}-\left(\bar{V}_{0}-v\right)^{\lambda-1}}{\lambda-1}\left(\left\langle\phi_{F}(v)\right\rangle-1\right) d v,
$$

a relation that we write, with obvious notations, as

$$
I(\lambda)=1
$$

Now we assume that $\phi_{F}>1$ is constant on its support. On the one hand, as $\lambda \rightarrow \phi_{F}$, the term $\int \frac{\bar{n}(v)}{\left(\bar{V}_{0}-v\right)^{\lambda}} d v$ tends to $+\infty$ and $\lambda-1 \rightarrow \phi_{F}-1$ for $v \approx \bar{V}_{0}$. Therefore,

$$
I(\lambda) \approx\left(\bar{V}_{0}\right)^{\lambda-1} \frac{V_{0}}{\overline{\mathcal{N}}_{0}} \int \frac{\bar{n}(v)}{\left(\bar{V}_{0}-v\right)^{\lambda}} d v \rightarrow+\infty \quad \text { as } \quad \lambda \rightarrow \phi_{F} .
$$

On the other hand, as $\lambda \rightarrow 0$,

$$
\bar{V}_{0} I(\lambda) \approx \frac{V_{0}}{\overline{\mathcal{N}}_{0}}-\phi_{F} \bar{V}_{0}^{\prime}+\bar{V}_{0}^{\prime}\left(\phi_{F}-1\right) \int \frac{\bar{n}(v)}{\bar{V}_{0}-v} d v
$$

Since $\bar{n}=\frac{\overline{\mathcal{N}}}{V_{0}\left(V_{0}-v_{1}\right)}\left(\bar{V}_{0}-v\right)$, Proposition 1 follows by the mean value theorem.

Numerical illustration. Numerical evidence, indicates that periodic solutions occur for the simple limiting model (6). We use the range of parameters produced in the previous subsection, when the firing rate $\phi_{F}$ is large enough and the nonlinear voltage $V_{0}(\mathcal{N})$ is stiff enough. The computed solution is depicted in the Figure 3 for the evolution equation (20). Here the choice of parameters and nonlinearities (which are far form the regimes where uniqueness of a steady state has been established) is given by the expressions

$$
\phi_{F}(v)=5 \mathbb{I}_{\{v>.7\}}, \quad V_{0}=.75+.22 \min (\mathcal{N}, 1)
$$



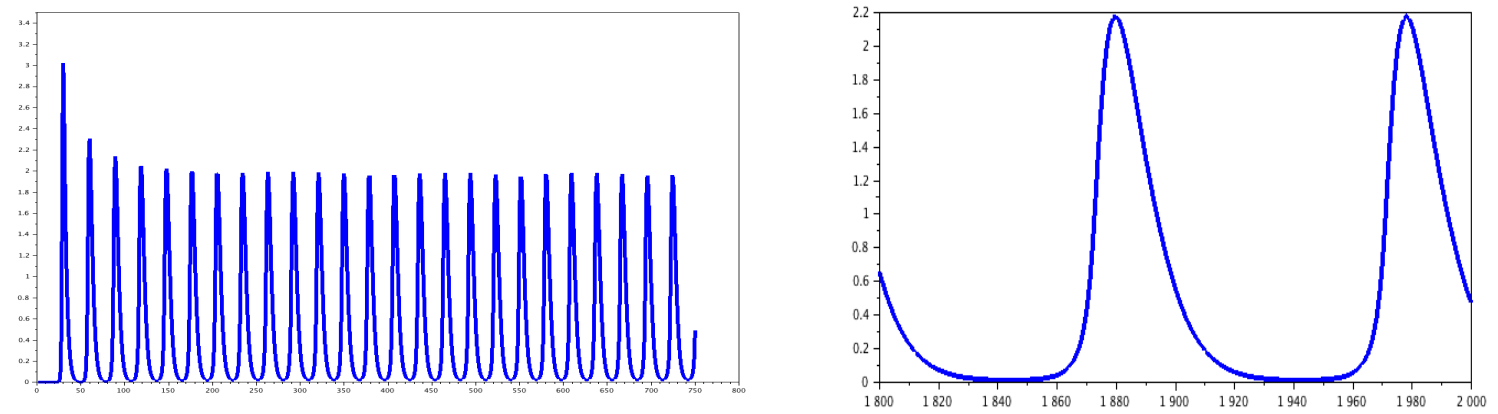

Figure 3: Numerical solutions of the evolution equation (20) with data in (34). Abscissae are $v$. Left: FIRING RATE $V_{0}(\mathcal{N})=.75+.15 \frac{\mathcal{N}}{.1+\mathcal{N}} ;$ Right: ZOOM ON TWO OSCILlations.

\section{Conclusion and perspectives}

Neural networks are by nature highly complex systems and mean field models are a way to circumvent the modeling. In [1, 2], the authors propose a kinetic mean field equation of neural assemblies to make a fine description of the dynamic of neural networks with respect to the membrane potential and the conductance of the neurons. The patterns that emerge from those models are very rich, and exhibit oscillations, bifurcations... However, this model is very difficult to tackle both from a numerical viewpoint [5], where efficient numerical schemes are complex to implement and from a theoretical viewpoint [6], where classical methods failed due to the particular boundary conditions and the degenerate diffusion involved in the equation. To overcome those difficulties, in [1, 2], the authors propose a reduction of dimension of this equation by closure moments. In this article, we give an alternative with a new kinetic model where the singular boundary condition is replaced by an integral absorption in order to obtain more precise theoretical results and to simulate the different types of dynamics that can emerge with a relatively simple algorithm on the limiting Integrate and Fire equation.

Several open questions about the present voltage only I\&F model remain open. The first one concerns a more precise theoretical study on the mechanisms beyond the oscillations in the Integrate and Fire model presented in this equation. Indeed, to our knowledge, there do not exists theoretical results of existence of periodic solutions for this kind of PDE. However, Equation (20) shares, in its structure, some similarity with the time elapsed model, where we can built explicit periodic solutions (see [12]). Indeed, as for the time elapsed model, in some regimes, the total firing rate satisfy a delay type equation, which may be tractable. As an example, considering for $k \in \mathbb{N}$ big enough (let us assume to simplify $V_{R}=0, V_{E}=1$ ) and the particular function

$$
\phi_{F}(v)=k \mathbb{I}_{v \geq \alpha}, \alpha>0, \quad 0<\alpha<1
$$

using the method of characteristics, we obtain the following formula on the total firing rate of Equation $(20)$

$$
\mathcal{N}(t)=k\left(1-\int_{\varphi(t)}^{t} \mathcal{N}(s) d s\right)
$$


with $\varphi(t) \leq t$ such that

$$
\alpha=e^{-(t-\varphi(t))} \int_{0}^{t-\varphi(t)} V_{0}(\mathcal{N}(s+\varphi(t))) e^{s} d s .
$$

This system is however more complex than the one obtained in [12], due to its specific coupling, but may be exploitable.

Coming back to the conductance and voltage kinetic model developed in [1, 2], a second important question, concerns the theoretical study of this equation which was initiated in [6]. Indeed, beside the question of well-posedness which is rather difficult, typical questions as the asymptotic convergence to a stationary state for very weak interconnections, is not yet understood.

The standard I\&F model for networks is closely related to (6) with two main differences. On the one hand firing is taken pointwise, rather than the nonlocal definition of firing in (6), which is our choice of a simplification. On the other hand noise is taken into account differently, directly by a diffusion term in the standard I\&F model, rather than by dispersion of the drift terms. It is written

$$
\left\{\begin{array}{l}
\frac{\partial}{\partial t} n+\frac{\partial}{\partial v}[h(b \mathcal{N}(t), v) n]-a \frac{\partial^{2} n}{\partial v^{2}}=\mathcal{N}(t) \delta\left(v-V_{R}\right), \quad t \geq 0, v<V_{E}, \\
n\left(V_{E}, t\right)=0, \quad \mathcal{N}(t)=-\left.a \frac{\partial n}{\partial v}\right|_{v=V_{E}} .
\end{array}\right.
$$

Another question is to derive this model from the voltage-conductance equation in a diffusive limit rather than an hyperbolic limit.

Acknowledgments BP has received funding from the European Research Council (ERC) under the European Union's Horizon 2020 research and innovation programme (grant agreement No 740623).

B.P. and D.S. acknowledge partial funding from the ANR blanche project Kibord ANR-13-BS01-0004 funded by the French Ministry of Research.

\section{References}

[1] D. Cai, L. Tao, M. Shelley, and M. D. W., "An effective kinetic representation of fluctuationdriven neuronal networks with application to simple and complex cells in visual cortex," PNAS, vol. 101, pp. 7757-7762, 2004.

[2] A. V. Rangan, D. Cai, and L. Tao, "Numerical methods for solving moment equations in kinetic theory of neuronal network dynamics," J. Comput. Phys., vol. 221, no. 2, pp. 781-798, 2007.

[3] N. Fourcaud and N. Brunel, "Dynamics of the firing probability of noisy integrate-and-fire neurons,," Neural Comp., vol. 14, pp. 2057-2110, 2002.

[4] W. Gerstner and W. M. Kistler, Spiking neuron models: Single neurons, populations, plasticity. Cambridge university press, 2002.

[5] M. J. Cáceres, J. A. Carrillo, and L. Tao, "A numerical solver for a nonlinear fokker-planck equation representation of neuronal network dynamics," J. Comp. Phys., vol. 230, pp. 10841099, 2011.

[6] B. Perthame and D. Salort, "On a voltage-conductance kinetic system for integrate \& fire neural networks," Kinet. Relat. Models, vol. 6, no. 4, pp. 841-864, 2013. 
[7] C. Villani, "Hypocoercivity," Mem. Amer. Math. Soc., vol. 202, no. 950, pp. iv+141, 2009.

[8] M. Herda and L. M. Rodrigues, "Large-Time Behavior of Solutions to Vlasov-Poisson-FokkerPlanck Equations: From Evanescent Collisions to Diffusive Limit," J. Stat. Phys., vol. 170, no. 5, pp. 895-931, 2018.

[9] J. Dolbeault, C. Mouhot, and C. Schmeiser, "Hypocoercivity for linear kinetic equations conserving mass," Trans. Amer. Math. Soc., vol. 367, no. 6, pp. 3807-3828, 2015.

[10] J. Pham, K. Pakdaman, J. Champagnat, and J.-F. Vivert, "Activity in sparsely connected excitatory neural networks: effect of connectivity," Neural Networks, vol. 11, pp. 415-434, 1998.

[11] K. Pakdaman, B. Perthame, and D. Salort, "Dynamics of a structured neuron population," Nonlinearity, vol. 23, no. 1, pp. 55-75, 2010.

[12] K. Pakdaman, B. Perthame, and D. Salort, "Relaxation and self-sustained foscillations in the time elapsed neuron network model," SIAM J. Appl. Math., vol. 73, no. 3, pp. 1260-1279, 2013.

[13] S. Mischler and Q. Weng, "Relaxation in time elapsed neuron network models in the weak connectivity regime," ACAP, 2018.

[14] D. Cai, L. Tao, A. V. Rangan, and D. W. McLaughlin, "Kinetic theory for neuronal network dynamics," Commun. Math. Sci., vol. 4, no. 1, pp. 97-127, 2006.

[15] M. J. Cáceres, J. A. Carrillo, and B. Perthame, "Analysis of nonlinear noisy integrate \& fire neuron models: blow-up and steady states," J. Math. Neurosci., vol. 1, pp. Art. 7, 33, 2011.

[16] J. A. Carrillo, M. d. M. González, M. P. Gualdani, and M. E. Schonbek, "Classical solutions for a nonlinear fokker-planck equation arising in computational neuroscience," Comm. in Partial Differential Equations, vol. 38, no. 3, pp. 385-409, 2013.

[17] J. A. Carrillo, B. Perthame, D. Salort, and D. Smets, "Qualitative properties of solutions for the noisy integrate and fire model in computational neuroscience," Nonlinearity, vol. 28, no. 9, pp. 3365-3388, 2015.

[18] F. Delarue, J. Inglis, S. Rubenthaler, E. Tanré, et al., "Global solvability of a networked integrateand-fire model of mckean-vlasov type," The Annals of Applied Probability, vol. 25, no. 4, pp. 20962133, 2015.

[19] G. Dumont and P. Gabriel, "The mean-field equation of a leaky integrate-and-fire neural network: measure solutions and steady states," ArXiv e-prints, Oct. 2017.

[20] N. Brunel and V. Hakim, "Fast global oscillations in networks of integrate-and-fire neurons with long firing rates," Neural Computation, vol. 11, pp. 1621-1671, 1999.

[21] P.-E. Jabin, "Macroscopic limit of Vlasov type equations with friction," Ann. Inst. H. Poincaré Anal. Non Linéaire, vol. 17, no. 5, pp. 651-672, 2000.

[22] J. Canizo and H. Yaldas, "Asymptotic behaviour of neuron population models structured by elapsed-time," Nonlinearity, In press. 
[23] P. Gabriel, "Measure solutions to the conservative renewal equation." working paper or preprint, Mar. 2018.

[24] V. Bansaye, B. Cloez, and P. Gabriel, "Ergodic behavior of non-conservative semigroups via generalized Doeblin's conditions." working paper or preprint, Sept. 2018.

[25] M. J. Cáceres and B. Perthame, "Beyond blow-up in excitatory integrate and fire neuronal networks: refractory period and spontaneous activity," J. Theoret. Biol., vol. 350, pp. 81-89, 2014. 